\title{
Photonic Calibration for Fluorescence Microscopy
}

\author{
Ian T. Young ${ }^{* a}$, Mohammed el Morabit ${ }^{\mathrm{a}}$, Guus Liqui Lung ${ }^{\mathrm{a}}$, Bart J. Vermolen, \\ ${ }^{a}$ Quantitative Imaging Group, Department of Imaging Science \& Technology, Delft University of \\ Technology, Lorentzweg 1, 2628 CJ Delft, The Netherlands; \\ ${ }^{\mathrm{b}}$ Biophysical Engineering Group, Faculty of Science and Technology, University of Twente, 7500 \\ AE Enschede, The Netherlands
}

\begin{abstract}
Based upon a collection of compact LEDs (light-emitting diodes) and a compact photodiode, we have developed a calibration tool for fluorescence microscopes that are used as digital imaging devices. The entire device (excluding a USB connector) measures $25 \mathrm{~mm}$ x $80 \mathrm{~mm}$ x $12 \mathrm{~mm}$. Virtually all commonly-used fluorophores can be simulated with one of the six LEDs. An LED is chosen from the host computer and its current range is selected (digitally) so as to provide a test of the complete dynamic range of the imaging system. Thus by varying the current through an LED in a controlled way, a controlled amount of "emission" light can be produced, transmitted through the chosen optical path of the microscope, and measured by the image sensor. The digitized intensity can then be determined as a function of the LED current. Any other (fluorescence) intensity measured through the same electro-optical path can then be characterized (and thus calibrated) by an equivalent electrical current.

The excitation light is calibrated by a photodiode which has a dynamic range of $10^{\wedge} 5: 1$ and thus is suitable for a variety of light sources: mercury lamps, lasers, LEDs, etc. The integration time of the photodiode as well as its gain can be digitally selected from the host computer. Further, using a Spectralon $^{\circledR}$ reflector, the inherent non-linearity of the LED emission versus current can be measured by the photodiode and used to provide a look-up table compensation independent of the image sensor used in the fluorescence microscope system.
\end{abstract}

Keywords: Fluorescence calibration, quantitative microscopy, LED slide, fluorescence microscopy

\section{INTRODUCTION}

In our previous publications and reports we have shown that it is possible to calibrate the "emission side" of a digital fluorescence microscope that is intended to be used as a quantitative measurement device ${ }^{1-3}$. The basic concept is quite straightforward. As illustrated in Figure 1, the two critical paths for calibration of an epi-illumination fluorescence microscope system are 1) the excitation path from the light source to the sample and 2) the emission path from the sample to the electro-optical sensor.

The emission path, including lenses, filters, mirrors, and the sensitivity of the sensor, can be calibrated using LEDs of varying colors placed at the location of the sample. The excitation path can be calibrated by placing a light sensor at the location of the sample. We have combined these two sensors into a single device whose dimensions are $25 \mathrm{~mm} \times 80 \mathrm{~mm}$ $\mathrm{x} 12 \mathrm{~mm}$. This device is shown in Figure 2 .

\footnotetext{
* i.t.young [at] tudelft [dot] nl; phone +31 15278 5390; www.ist.tudelft.nl/qi
} 

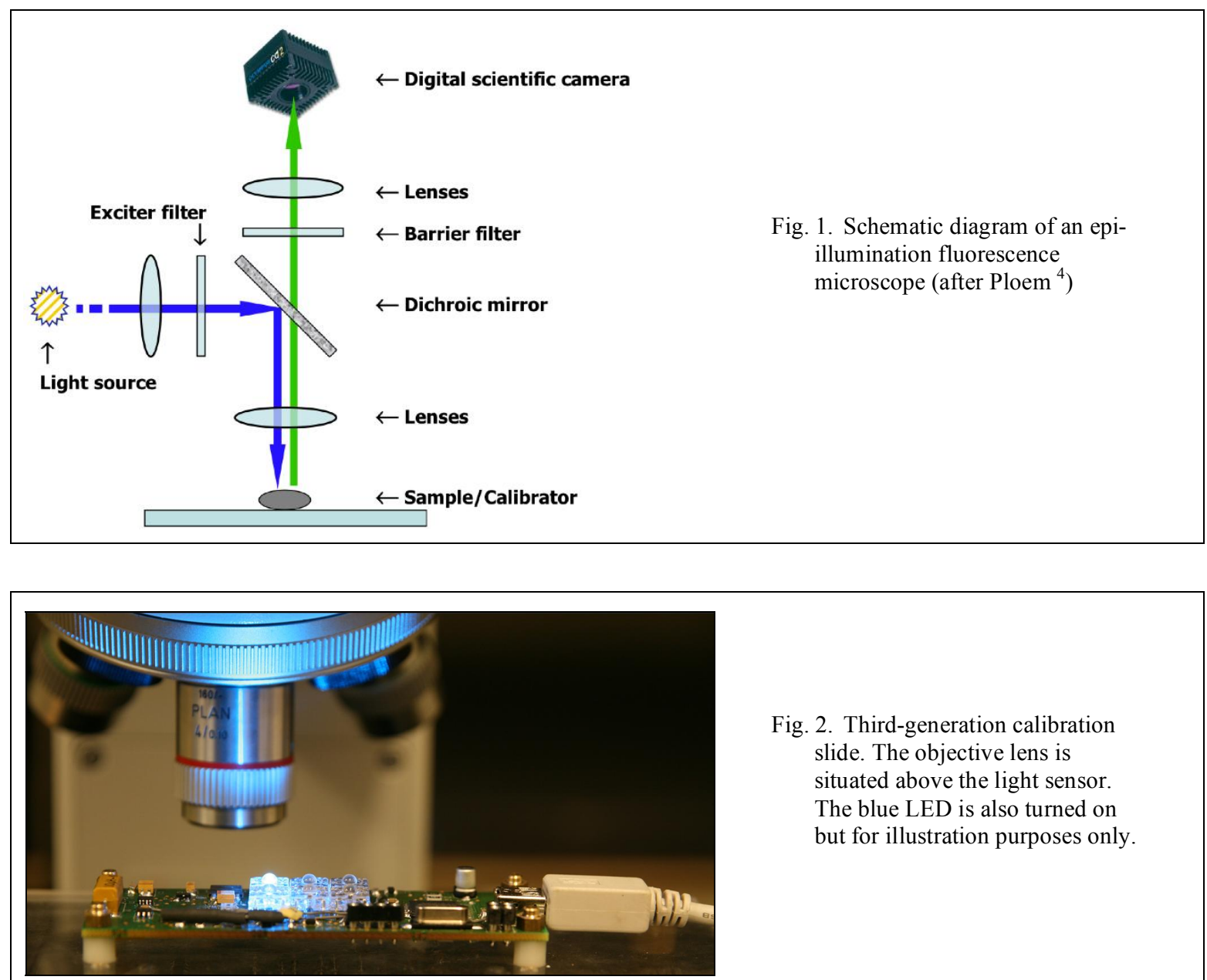

Fig. 2. Third-generation calibration slide. The objective lens is situated above the light sensor. The blue LED is also turned on but for illustration purposes only.

\subsection{The emission path}

Low-power light-emitting diodes (LEDs) are available in a number of colors. An LED color mimics the emission spectrum of a fluorophore and each LED spectrum is relatively narrow band. The specific LEDs that we use in our device are listed in Table 1 and their "emission" spectra are shown in Figure 3. The narrowest spectrum is found in the amber LED (ML00) with a measured full-width, half-maximum (FWHM) of $21 \mathrm{~nm}$ and the widest spectrum is in the green LED (MG00) with a measured FWHM of $47 \mathrm{~nm}$.

Table 1. The six LEDs used in the system described in this paper. The indicated wavelengths correspond to the peak wavelengths measured for each LED.

\begin{tabular}{|l|l|l|l|}
\hline \multirow{2}{*}{ LEDs } & $\begin{array}{l}477 \mathrm{~nm}-\text { blue LumiLed } \\
\text { DS05 HPWN MB00 }\end{array}$ & $\begin{array}{l}514 \mathrm{~nm}-\text { cyan LumiLed } \\
\text { DS05 HPWN MC00 }\end{array}$ & $\begin{array}{l}550 \mathrm{~nm}-\text { green LumiLed } \\
\text { DS05 HPWN MG00 }\end{array}$ \\
\hline \multirow{2}{*}{ LEDs } & 591 nm - amber LumiLed & $619 \mathrm{~nm}-$ orange LumiLed & $633 \mathrm{~nm}-$ red LumiLed \\
& DS05 HPWT ML00 & DS05 HPWT MH00 & DS05 HPWT MD00 \\
\hline
\end{tabular}

We used an ASI spectral imager (Applied Spectral Imaging, Migdal Ha'emek, Israel), to measure the spectrum $E(\lambda)$ and to determine the peak wavelength, the average wavelength, and the spectral width for each LED type. A $500 \mathrm{~nm}$ narrowband interference filter was used to check the readout of the spectral imager. 


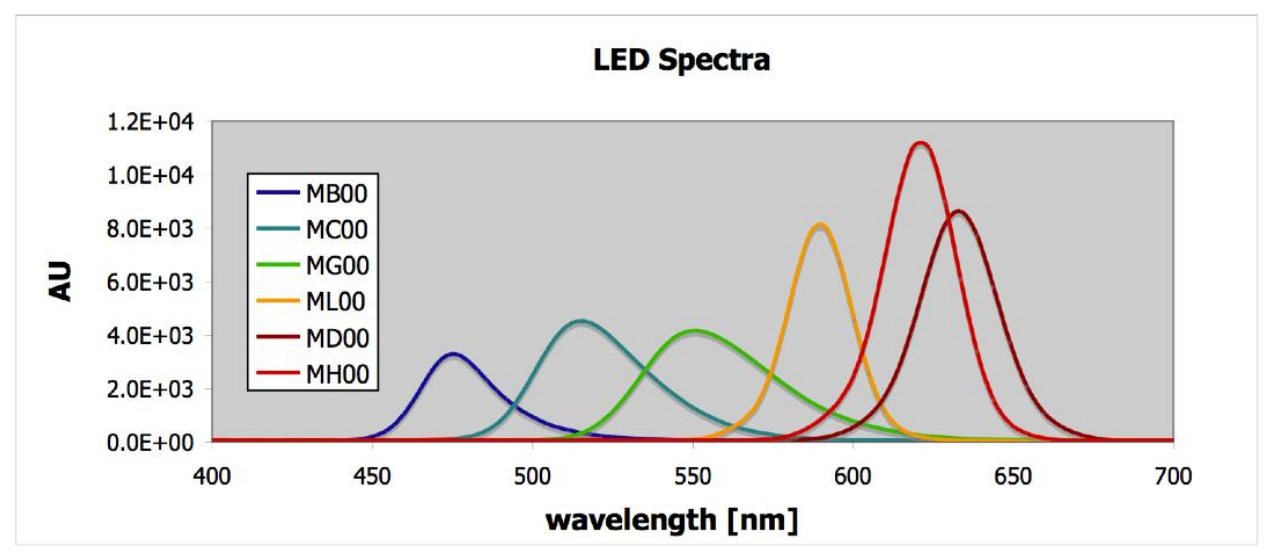

Fig. 3. Six LEDs are used in our calibration slide. Their measured spectra are shown here.

The actual peak intensity of each LED can vary considerably from one color to another. The highest peak intensity was measured for the orange LED (MH00) at $\lambda=619 \mathrm{~nm}$ and a value of $1.1 \times 10^{4}$ [AU] and the lowest peak intensity was measured for the blue LED (MB00) at $\lambda=477 \mathrm{~nm}$ and a value of $3.3 \times 10^{3}$ [AU], representing a dynamic range in peak values of 3.3 .

We also observed that the brightness intensity of the LED does not, in general, increase linearly with input current. The behavior is similar to an "S-curve" with different regions: lag, onset, linear, asymptotic, and saturation. This is shown in Figure 4.

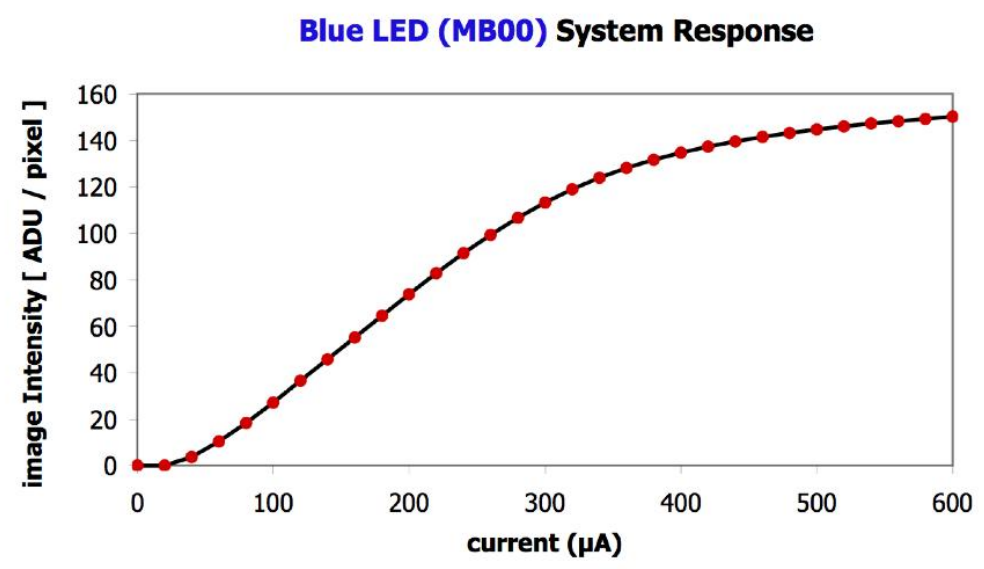

Fig. 4. Response of a microscope system to the blue LED ${ }^{2}$.

While some of this behavior is due to components in the system other than the LED (the camera, for example), the basic shape of the curve is characteristic of LEDs. As we will describe below, this curve can be measured for each LED and corrected for by use of a simple lookup table.

\subsection{The excitation path}

Characterization of the excitation path means knowing how much of the light provided by the illumination source (mercury lamp, xenon lamp, halogen lamp, laser, or high-power LED $^{1}$ ) actually reaches the sample. This can vary as a light sources ages, as microscope objectives are changed, or as different filter packs are used. Our sensor is a photodiode system embedded in a sophisticated chip. The system is the Intersil ISL 29004 Light-to-Digital Output Sensor (Intersil Corp., Milpitas, California, www.intersil.com).

The photodiode chip contains not only the photodiode element but also a 16-bit analog-to-digital converter (ADC), the ability to choose from four different measurement ranges in the ratios 1000:4000:16000:64000, and the ability to choose 
the integration time for measurement. There is built-in $50 \mathrm{~Hz}$ and $60 \mathrm{~Hz}$ noise rejection and the power and voltage requirements are compatible with use in USB-based instrumentation. The dimensions of the chip are $2.8 \mathrm{~mm} \times 2.2 \mathrm{~mm} \times$ $0.7 \mathrm{~mm}$.

\section{EMBODIMENT}

This system has evolved over the past three years. The first version involved eight LEDs to calibrate the emission path. The intention was to calibrate the excitation path (filters plus a mercury lamp, laser, or high-power LED ${ }^{1}$ ) using fluorescent plastic material as standards. These relatively inexpensive sheets can be cut into microscope slide format (75 $\mathrm{mm} \times 25 \mathrm{~mm}$ ) to serve as surrogate specimens that are sensitive to neither photobleaching nor aging. Examples are shown in Figure 5.

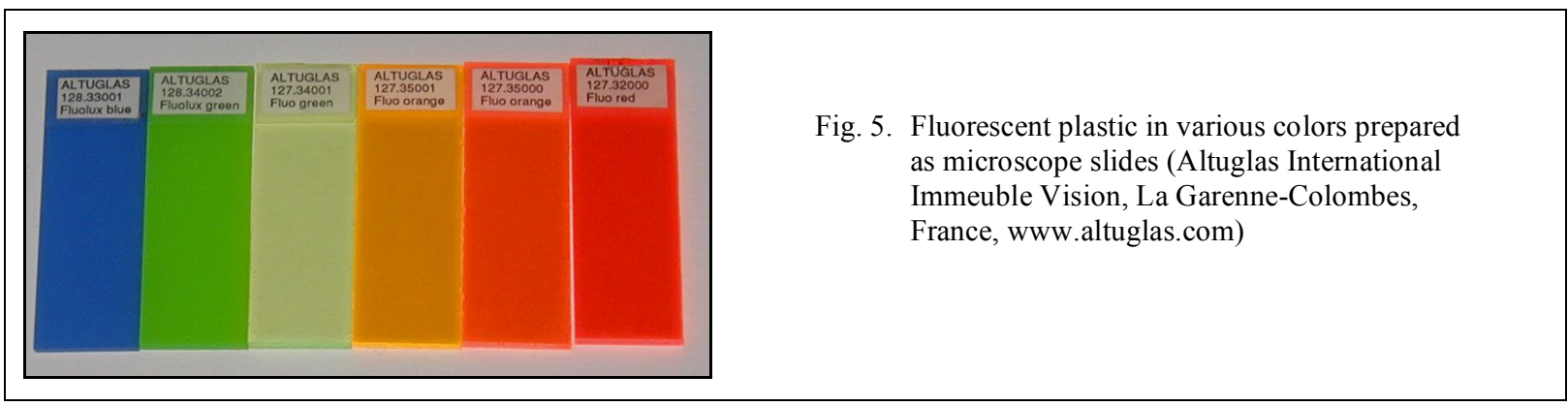

Unfortunately, the concentration of fluorescing molecules in the plastic is so high that they cannot be used as calibration samples. The fluorescence emission remains constant across a range of useful excitation intensities making them unsuitable.

The second version involved the use of a TAOS TSL2561 Light-to-Digital Converter (Texas Advanced Optoelectronic Solutions Inc., Plano, Texas, www.taosinc.com). This chip-based system should have been appropriate but there appeared to be on-chip design errors that made it inappropriate as a sensing device in our application. We were unable to obtain a monotonically decreasing output value when the chip was exposed to a monotonically decreasing amount of input light,

The third version of the calibration slide uses the Intersil chip instead of the TAOS chip and satisfactory measurement results that will be described below. This embodiment is illustrated in Figure 6. It is clear from Figure 6 that there is only room for six LEDS and these correspond to the six given in Table 1.

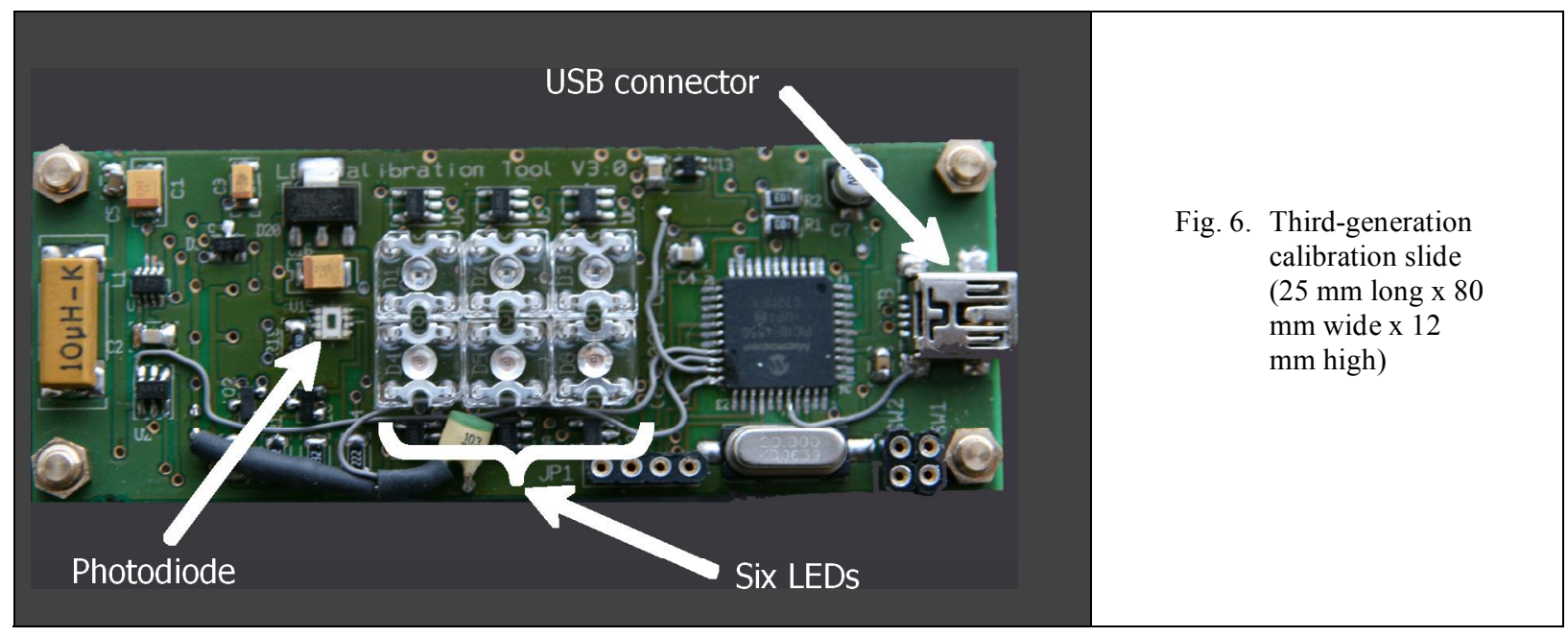




\section{MEASUREMENTS}

We have already demonstrated the efficacy of the LEDs as surrogate fluorescing cells ${ }^{2,3}$. In this section we will present the measurements that have been made with the Intersil photodiode chip. We have used a $100 \mathrm{~W}$ mercury lamp on a Leitz Aristoplan microscope with a 4x/NA 0.1 lens. A DAPI filter set was used thus determining the exciter filter, the dichroic mirror, and the barrier filter. (See Figure 1.)

\subsection{Excitation intensity calibration}

A set of three neutral density (ND) filters used singly and in combination was used to determine the measurement characteristics of the photodiode. The values of the filters were listed as ND2 $=1 / 2=50 \%, \mathrm{ND} 4=1 / 4=25 \%$, and ND32= $1 / 32=3.1 \%$. The basis for comparison was an Ophir Nova Laser Power Monitor (Ophir Optronics, Jerusalem, Israel, www.ophiropt.com) set for a wavelength measurement of $450 \mathrm{~nm}$. The results are shown in Figure $7 \mathrm{a}$.

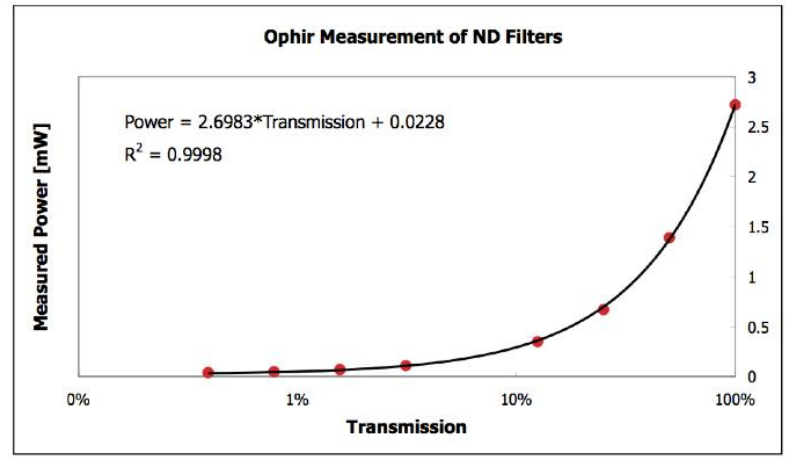

Fig. 7a. Characterization of the ND filters from the light source, through the DAPI filters and the objective lens, to the Ophir photosensor

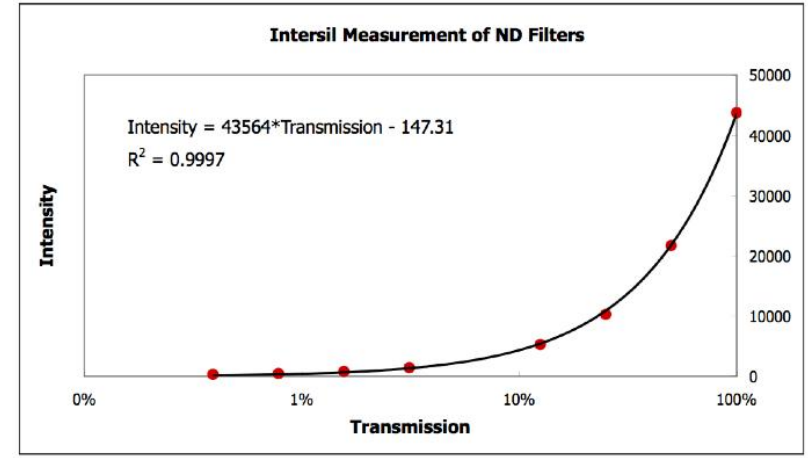

Fig. 7b. Characterization of the ND filters using the Intersil photosensor

When the same experiment is repeated but using the Intersil sensor the results are shown in Figure $7 \mathrm{~b}$. Our implementation has selectable integration times from $5 \mathrm{~ms}$ to $100.7 \mathrm{~ms}$ and the integration time used here was $47.9 \mathrm{~ms}$ with the range set for 64000. The agreement between the two sensors is excellent and a comparison, shown in Figure 8, indicates that the Intersil photodiode chip is suitable for this application.

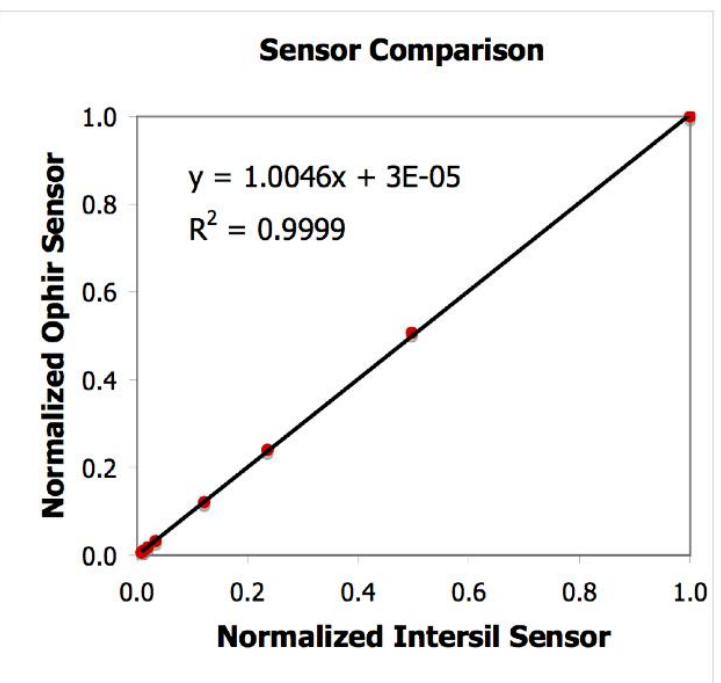

Fig. 8. Correlation between Ophir sensor and Intersil sensor. The data sets from Figures 7a,b were first normalized for this comparison. 


\subsection{Integration time calibration}

As it is possible to adjust both the integration time and the amplification range of the sensor, it is important to demonstrate that the sensor is calibrated across the spectrum of possible values of these two parameters. Figure 9a-d shows four such measurements where different ND filter combinations were used with different range settings (as indicated) and the intensity measurements were then made for varying integration times. The maximum value of the output is 65535 due to the 16-bit ADC.

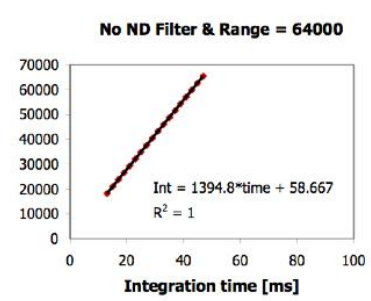

Fig. 9a. No ND filter was used and the range was set to 64000. The integration was then varied from $15 \mathrm{~ms}$ to $46.9 \mathrm{~ms}$.

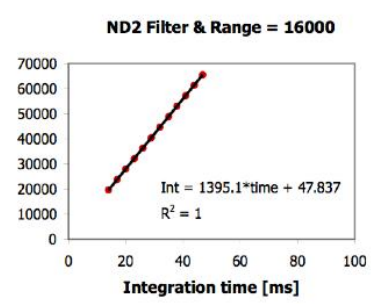

Fig. 9b. An ND2 filter was used corresponding to $50 \%$ transmission and the range was set to 16000 .

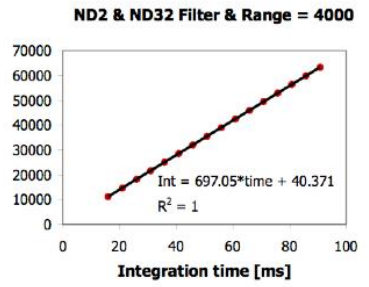

Fig. 9c. A combination of ND2 \& ND32 filter was used corresponding to $1.6 \%$ transmission and the range was set to 4000 .

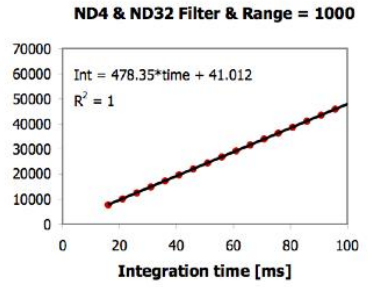

Fig. 9d. A combination of ND4 \& ND32 filter was used corresponding to $0.8 \%$ transmission and the range was set to 1000 .

It is clear that the sensor is linear in all cases. The maximum value in Fig. 9a is 65495 and that is reached with an integration time of $46.9 \mathrm{~ms}$. Figures $9 \mathrm{~b}$ indicates that when the intensity decreases by a factor of four (ND2 filter) but the range changes by the same factor of four (from 64000 to 16000) then the time required to reach an intensity of 65488 is the same, $46.9 \mathrm{~ms}$.

\subsection{Internal LED calibration}

In section 1.1 we indicated that the light emission from an LED was not linear across all current values and we illustrated that in Figure 4. To characterize this departure from linearity without using an external sensor (which might suffer from its own non-linearity), we have devised a procedure using the photodiode chip and a reflecting cover made of Spectralon $^{\circledR}$ (Labsphere, North Sutton, New Hampshire, www.labsphere,com) to measure the LED light-versus-current characteristics. This modification is shown in Figure 10 for two different LEDs.

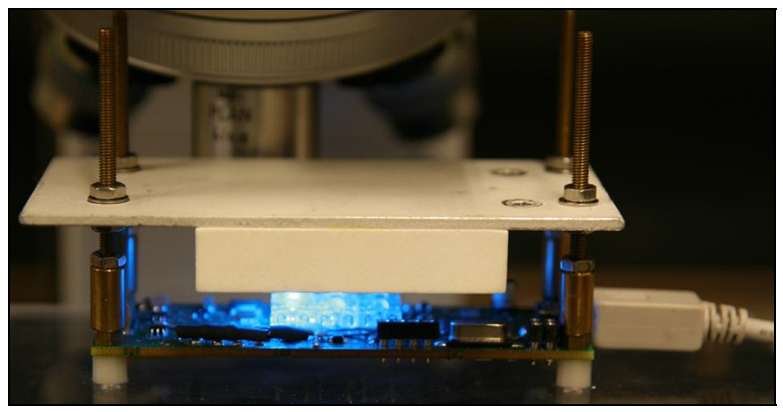

Fig. 10a. Spectralon ${ }^{\circledR}$ block is directly above the LEDs and the Intersil photodiode. (See Fig. 6) The blue LED is illuminated and light is reflected to the photodiode.

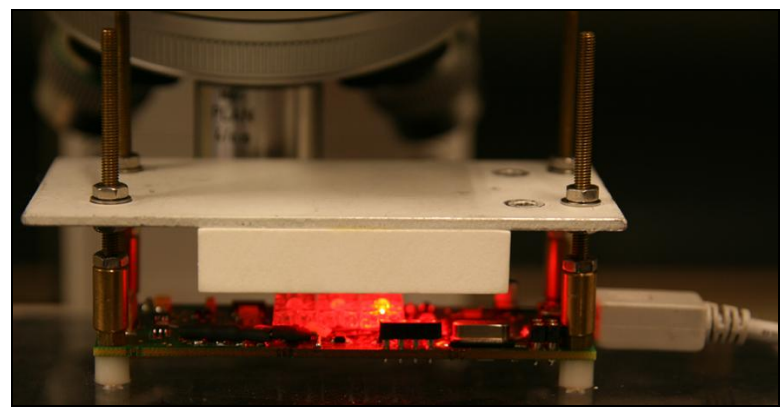

Fig. 10b. The red-orange LED is illuminated and light is reflected to the photodiode. Notice the longer light path from the LED to the photodiode sensor.

Using the configuration illustrated above we measured the light-versus-current characteristics of the various LEDS. The data shown in Figure 11 were measured with range $=1000$ and integration time $=100.7 \mathrm{~ms}$. The blue LED produces 
more than $12 \mathrm{x}$ the intensity of the red-orange LED at the sensor. This is due to the different lengths of the light paths from the LEDs to the photodiode. This, however, is not important as we are interested in measuring the shape of the light-versus-current curve as opposed to its absolute values.

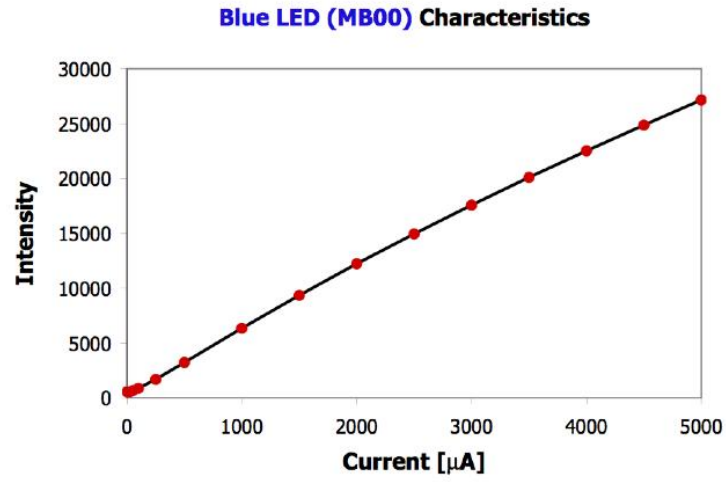

Fig. 11a. Light intensity from the blue LED reflected off the Spectralon ${ }^{\circledR}$ block to the Intersil photodiode as a function of LED current.

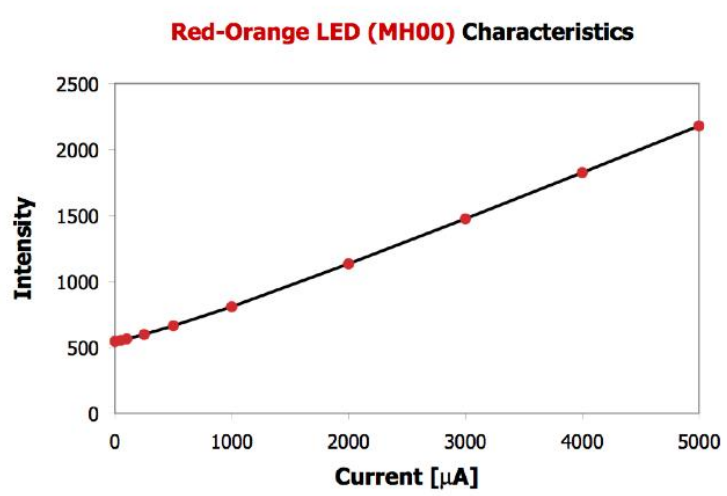

Fig. 11b. Light intensity from the red-orange LED as a function of LED current.

These curves can be used as look-up tables (reference curves) to determine the actual current associated with any given light level and this in turn can be used for the calibration of the emission side of the fluorescence microscope as described in our earlier publication ${ }^{2,3}$.

\section{SUMMARY AND CONCLUSIONS}

We have developed a system that allows calibration of both the excitation and emission side of an epi-illumination fluorescence microscope. This system is:

- $\quad$ small - The dimensions are $25 \mathrm{~mm}$ x $80 \mathrm{~mm}$ x $12 \mathrm{~mm}$.

- portable - The device runs off the USB port of any modern computer.

- inexpensive - The parts cost approximately $€ 250$

- easy-to-use - The device can be used from either a program such as ZTerm (for Apple Macintosh), HyperTerminal (for Windows machines), or MatLab.

- precise - We have not shown error bars for the graphs in this paper because they are too small to be seen. Every measurement presented was the average of a number of measurements and typical coefficients-ofvariation $(C V=100 \% * \sigma / \mu)$ are less than $0.2 \%$.

We expect that future developments will be modifying the system so that it will be suitable for inverted microscopes and various environmental conditions. Further, we are working to make the software even more "user friendly" including auto-ranging.

\section{ACKNOWLEDGMENTS}

This work was partially supported by the Bsik Research Program Cyttron and the Bsik Research Program MicroNed.

\section{REFERENCES}

${ }^{1}$ I.T. Young, Y. Garini, H.R.C. Dietrich et al., "LEDs for Fluorescence Microscopy," presented at the ThreeDimensional and Multidimensional Microscopy: Image Acquisition and Processing XI, San Jose, California, J.-A. Conchello, C. Cogswell, and T. Wilson ed., Vol. 5324, pp. 208-215, Publisher SPIE, 2004. 
2 I.T. Young, Y. Garini, B. Vermolen et al., “Absolute Fluorescence Calibration,” presented at the Imaging, Manipulation, and Analysis of Biomolecules, Cells, and Tissues IV, San Jose, California, Daniel L. Farkas, Dan V. Nicolau, and Robert C. Leif ed., Vol. 6088, pp. 1-9, Publisher SPIE, 2006.

${ }^{3}$ I.T. Young and G. Liqui Lung, "LEDs and photodiodes as calibration standards for microscopy," in Focus on Microscopy (Valencia, Spain, 2007).

${ }^{4}$ J.S. Ploem, "The use of a vertical illuminator with interchangeable dichroic mirrors for fluorescence microscopy with incident light," Zeitschrift für wissenschaftliche Mikroscopie, Z. Wiss. Mikr., 68, pp. 129-142, 1967. 